\title{
Light emission through a corrugated metal film: The role of cross-coupled surface plasmon polaritons
}

\author{
S. Wedge, ${ }^{1}$ I. R. Hooper, ${ }^{1}$ I. Sage, ${ }^{2}$ and W. L. Barnes ${ }^{1}$ \\ ${ }^{1}$ Thin Films Photonics Group, School of Physics, Stocker Road, University of Exeter, Exeter, EX4 4QL, United Kingdom \\ ${ }^{2}$ Photonics and Displays Group, QinetiQ, St. Andrews Road, Malvern, Worcestershire, WR14 3PS, United Kingdom
}

(Received 24 February 2004; published 28 June 2004)

\begin{abstract}
We examine the phenomenon of light emission through a thin metal film that takes place via surface plasmon polaritons. Surface plasmon polariton cross coupling has recently been invoked to explain sharp features observed in the angle dependent emission spectra obtained from surface-emitting (through cathode) organic light-emitting diode structures. We investigated whether such a cross-coupling process is needed to explain such observations. We undertook measurements on samples for a variety of metal film thicknesses. Our results are consistent with the mechanism of surface plasmon polariton cross coupling but also show that the processes underlying the emission from such structures can be rather subtle.
\end{abstract}

DOI: 10.1103/PhysRevB.69.245418

PACS number(s): 78.66.Bz, 85.60.Jb, 42.79.Dj

\section{INTRODUCTION}

There is continuing interest in the emission of light from surface-emitting organic light-emitting diodes (OLEDs) $)^{1,2}$ since such devices might be integrated onto opaque substrates, for example, in actively addressed display applications. In common with the more usual substrate-emitting OLED, not all of the light emitted in the organic layer emerges since much of it is trapped by guided modes of the OLED structure, including surface plasmon-polariton (SPP) modes associated with the cathode/organic interface. ${ }^{3,4}$ Various strategies have been adopted to recover some of this lost power in substrate-emitting OLEDs so as to improve device efficiency, among them the use of wavelength scale periodic microstructure to scatter guided modes to radiation. ${ }^{5}$ Surface-emitting OLEDs have similar problems that are arguably even more complex since the thin metallic cathodes used in these devices support two SPP modes. Emitters in the organic layer may couple to these modes; one mode is associated with the cathode/organic interface, the other is associated with the cathode/air interface. Gifford and Hall ${ }^{6}$ recently discussed the use of microstructure to recover these SPP modes through a surface plasmon polariton cross-coupling process in which the microstructure is first used to couple the SPP modes associated with the two interfaces together, the same microstructure is then also used to provide coupling of the SPP modes to light. Here we report the results of an investigation on a range of similar structures designed to explore this cross-coupling process in more detail.

Surface plasmon polariton cross coupling was first reported by Pockrand in 1975 (Ref. [7]) through the use of reflectivity measurements on thin corrugated metal films. SPP cross-coupling was discussed theoretically by Weber and Mills, ${ }^{8}$ and there followed a number of further reports. ${ }^{9,10}$ Ushioda et al. ${ }^{11}$ invoked roughness mediated cross-coupling to explain some of their results on light emitting tunnel junctions but it was Gruhlke et al. ${ }^{12}$ who first reported the emission of light generated by molecular fluorescence through a corrugated metal film mediated by SPP cross coupling in 1986. The transmission of light through nanostructured metallic films received renewed interest following the report in 1998 by Ebbesen and co-workers ${ }^{13}$ of enhanced transmission through metallic films perforated with periodic arrays of subwavelength holes. In the context of the work reported here the question of whether such enhanced transmission requires an array of holes or can be accomplished with just a periodically modulated film is of particular interest. ${ }^{14}$ Several authors have explored the possibility of using periodically modulated metal films to extract light from surface plasmon polariton modes, ${ }^{15-20}$ though only Gifford and Hall ${ }^{6,21}$ have so far reported experimental results specifically to look at surface plasmon polariton cross coupling as a means of extracting light from such structures.

In a surface-emitting OLED light has to traverse the thin metallic cathode in order to emerge into the far field, as shown in the inset to Fig. 1(a). In such structures the cathode is bound on one side by the emissive organic layer, and on the other by air. Consequently two distinct SPP modes may be supported by the structure, one associated with each metal/dielectric interface. Figure 1(a) shows a schematic dispersion diagram, angular frequency $\omega$ vs in-plane wave vector $k_{\|}$(here in-plane refers to the plane defined by the interfaces of the structure and, where it refers to emission, $k_{\|}$is also in the plane containing the emission direction) for a thin planar metal film bounded on one side by an organic layer and on the other by air. The shaded area in Fig. 1(a) is the air light-cone, it represents those combinations of frequency and in-plane wavevector applicable to freely propagating photons in the air half space. As can be seen in Fig. 1(a), the wave vectors of the two SPP modes are always greater than that of a photon of the same frequency in air, reflecting the nonradiative nature of SPPs. ${ }^{22}$ If nothing is done to recover the energy in these modes they represent an unwanted loss mechanism.

As noted above, one method of recovering some of this energy is to corrugate the metal surface on the scale of the emission wavelength with period $\lambda_{g}$, as shown in the inset to Fig. 1(b). The momentum (wave vector) of the SPPs may then be augmented or reduced by grating scattering off the periodic structure according to the relation 

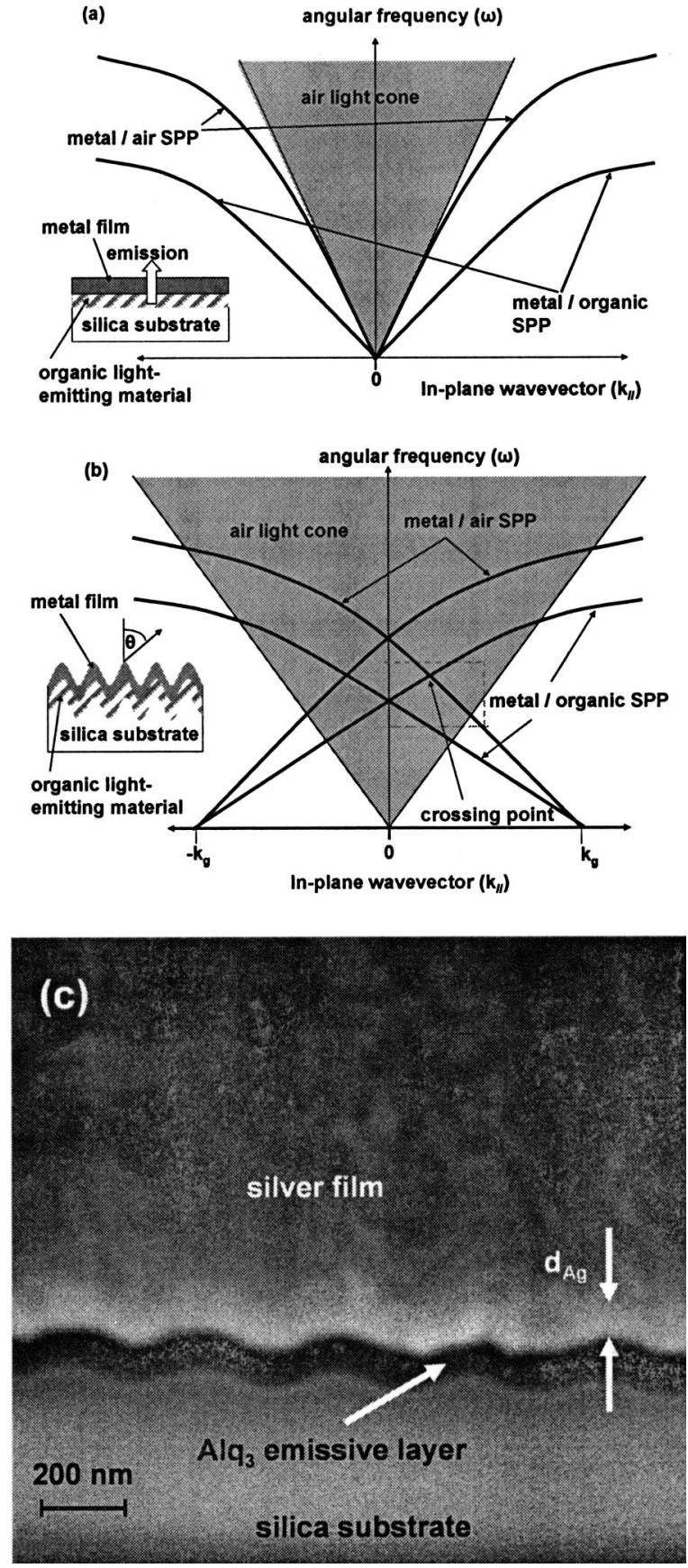

FIG. 1. (a) Schematic representation of a dispersion map for a thin metal film bordered on one side by an organic light-emitting material and on the other side by air (inset). The shaded region labeled air light-cone represents the frequencies and wave vectors accessible to light propagating in air. (b) Schematic representation of a dispersion map for a corrugated thin metal film bordered on one side by an organic light-emitting material and on the other side by air (inset). The area enclosed within the dashed lines represents the range of frequencies and wave vectors presented in Fig. 4. [Note that for clarity the horizontal scale used in (b) not the same as (a).] (c). An SEM of the sample with a silver film thickness of $61 \mathrm{~nm}$. The image was obtained by first milling a trench in the sample using a focussed ion-beam milling machine (FEI DB2000). The image is taken at an angle of $52^{\circ}$ from the normal, courtesy of E. Devaux and T. Ebbesen, ISIS, Strasbourg, France.

$$
k_{\mathrm{SPP}}^{\prime}=k_{\mathrm{SPP}} \pm n k_{g}
$$

where $k_{\mathrm{SPP}}$ is the wave vector of the SPP mode, $k_{g}$ is the grating wave vector $\left(k_{g}=2 \pi / \lambda_{g}\right), k_{\mathrm{SPP}}^{\prime}$ is the in-plane wave vector of the scattered SPP mode, and $n$ is an integer that defines the order of the scattering process. Through an appropriate choice of grating period, first-order scattering may allow the SPP modes to be coupled to radiation, i.e., $\left|k_{\text {SPP }}^{\prime}\right|<k_{0}$, where $k_{0}=\omega / c$ is the free space wave vector. The effect of first-order grating scattering is shown on the dispersion diagram depicted in Fig. 1(b). Coupling to far-field light is possible where scattering has placed a mode within the air light cone. Using grating coupling in this way opens a route for light trapped within a surface-emitting OLED device to be recovered.

An interesting feature associated with the grating scattered dispersion curve can be noted in Fig. 1(b). For a certain $\omega, k_{\|}$combination the positively (negatively) scattered SPP mode associated with the metal/air interface is degenerate with the negatively (positively) scattered SPP mode associated with the metal/organic interface. It is emission corresponding to this $\omega, k_{\|}$point that was the subject of the investigation by Gifford and Hall; ${ }^{6}$ we will refer to this $\omega, k_{\|}$value as the crossing point. At the wavelength and emission angle corresponding to the $\omega, k_{\|}$crossing point emission mediated by the metal/air and metal/organic SPP coincide, one thus expects emission at this point to be significantly greater than emission at neighboring wavelengths/angles. For the samples studied by Gifford and Hall a significant interaction between these modes was invoked to explain their data. We wished to further explore the possibility of SPPs cross coupling in the context of light emitting structures, with a view to probing the underlying mechanism. We did this by looking at emission through a number of corrugated thin metal films of different thickness.

Before going further it is useful to review the nature of the SPP modes associated with a thin metal film. If the metal film that supports the SPP modes is thin enough then the fields associated with the modes of each interface may overlap. If these two modes also have the same wave vector $k_{\|}$for a given angular frequency $\omega$, then the two modes are degenerate and there is a possibility that they will interact. Such a situation occurs for the symmetrically clad metal film (i.e., the materials on either side of the metal have the same refractive index); the interaction leads to a lifting of the degeneracy of the two modes to produce two coupled surface plasmon polariton modes whose fields span across the metal film. ${ }^{23-26}$ In an asymmetric system, such as the structures studied here, in which the media bounding the metal have different refractive indices, the interaction described above cannot take place because the two single interface SPPs are no longer degenerate, for any given frequency they always have different in-plane wave vectors. Coupling of these modes is now only possible through a scattering process that allows this wavevector difference to be overcome, such as that offered by a grating. When the coupling between the two SPP modes is mediated by the grating in this way the following Bragg condition must be satisfied. 


$$
\pm k_{\mathrm{SPP} \text { org }} \mp 2 k_{g}=\mp k_{\mathrm{SPP} \text { air }} \text {, }
$$

where, $k_{\mathrm{SPP} \text { org }}$ and $k_{\mathrm{SPP} \text { air }}$ are the wave vectors associated with the SPPs at the metal/organic and metal/air interfaces respectively.

We note that two regimes of cross coupling are possible in the asymmetric structures considered here; in both cases the grating may allow energy from the SPP mode on one interface to be transferred to the SPP mode on the second interface. First, the coupling is weak so that the dispersion of the individual modes remains unaltered. Second, if conditions were such as to allow a stronger coupling between the SPP modes, e.g., with a deeper grating, then one might expect the degeneracy of the two modes to be lifted. In this later case one expects that the dispersion of the modes will be altered, in particular the interaction should result in an anticrossing feature in the dispersion diagram at the crossing points of the two modes; $;^{7}$ as we will see, although we find evidence for cross coupling we did not observe such an anti-crossing features and thus conclude that in our structures the first regime applies.

Summarizing the role of the periodic structure, in the cross-coupling process the metal/organic SPP is first coupled to the metal/air SPP by scattering, which in turn is coupled to radiation, again by scattering. Both of these processes rely on scattering from the grating, the first requires second-order scattering (i.e., by $2 k_{g}$ ) while the coupling of the metal/air SPP to radiation requires first-order scattering $\left(k_{g}\right)$. As we will see, this simple view needs to be modified once a better understanding of the details of the SPP mediated emission process has been acquired.

At this point it is instructive to see how well emitters located within the light-emitting layer couple to the two different SPP modes. We made use of a well established theoretical model to undertake such calculations. ${ }^{27,28}$ The emitters are modeled as damped electric dipole sources, reflections from the interfaces acting to drive the sources, thus modifying their emission. Using such a model one can calculate the power lost by a source as a function of in-plane wave vector, known as the power dissipation spectrum. One example of such a power dissipation spectrum is shown in Fig. 2(a). We have chosen an emission wavelength of $550 \mathrm{~nm}$ and taken the relative permittivity of the silver to be $-11.0+0.3 i$ and the silver film thickness to be $30 \mathrm{~nm}$. (In addition we have assumed the dipole to have an isotropic orientation, i.e., the dipole moment associated with the emission of light samples all directions in space on a time scale faster than the luminescence lifetime.)

The peaks in such a spectrum indicate power lost by the emitter to modes of the structure. It is striking in Fig. 2(a) that while power coupled to the SPP mode associated with the metal/organic interface is very substantial, that associated with the metal/air interface is very small, down by over a factor of 100. This finding is very important since it shows that if one is to extract the power lost to the SPP modes of structures such as those considered here it is the SPP associated with the metal/organic interface upon which our attention should be focussed, not the SPP associated with the metal/air interface; it is this latter mode which has more fre-
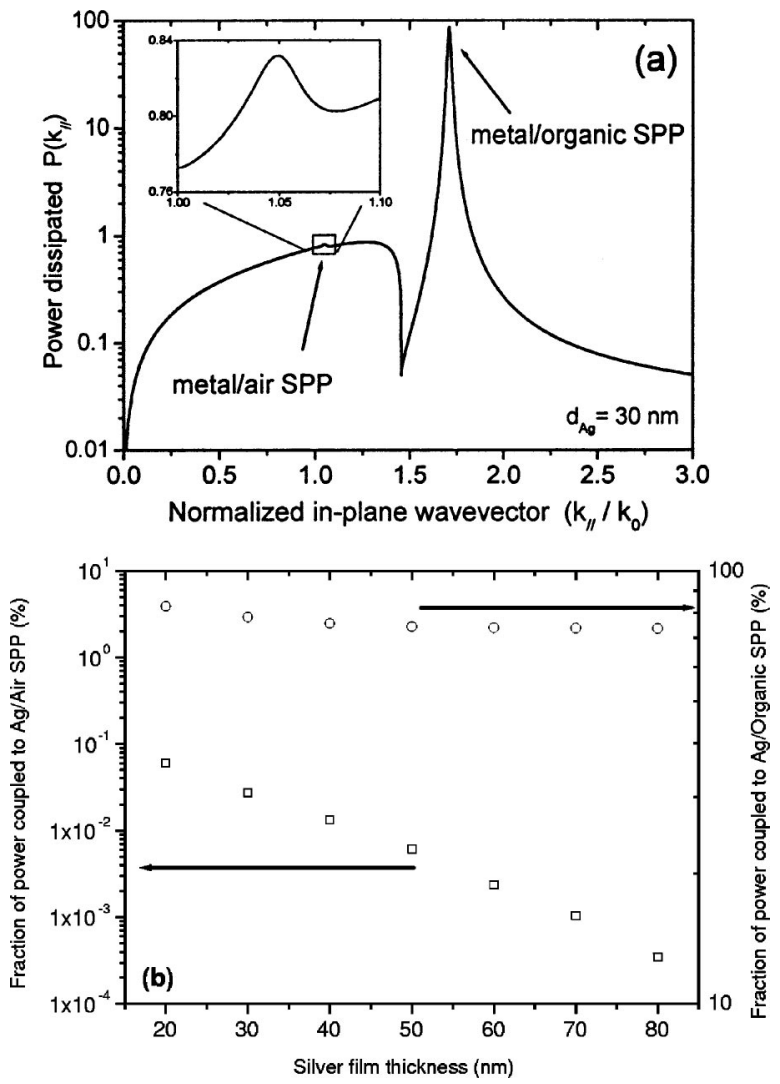

FIG. 2. Calculated power dissipation spectrum on a logarithmic scale for an emitter located in the light-emitting at a distance of $20 \mathrm{~nm}$ from the metal surface (a). The large peak at a normalized in-plane wave vector of $\sim 1.7$ indicates power being lost to the metal/organic SPP, the inset is a expanded plot (linear scale) of the feature associated with the metal/air SPP. The power coupled to the metal/organic and metal/air SPP modes as a function of silver film thickness (b).

quently been investigated in the past. ${ }^{15,16,19,20}$

We can extend the value of such calculations by integrating the area under each peak in the power dissipation spectrum. By so doing we can determine the power coupled to each mode as a fraction of the total power radiated by the emitter. We have done this as a function of silver film thickness, the results are shown in Fig. 2(b). The coupling to the metal/organic SPP is largely independent of silver film thickness whilst the coupling to the metal/air SPP falls exponentially with silver film thickness, much as one might have expected. These calculations will be discussed below when we come to interpreting our experimental data. (Note that in these calculations we assumed the quantum efficiency of the emitter to be unity.)

\section{EXPERIMENTAL}

We started our investigation by looking at the photoluminescence (PL) from structures similar to those shown schematically in the inset to Fig. 1(b). In the first instance we wished to establish the nature of the modes supported by such structures, something that we accomplished by measur- 
ing the emission spectra for a range of emission angles so as to build up a dispersion diagram. Below we describe both the fabrication of our samples and the techniques used to acquire the dispersion data.

\section{A. Sample fabrication}

Samples were made on corrugated silica substrates, the grating profiles being produced by exposing a silica substrate coated with a photoresist film (Shipley Megaposit SPR 7001.2) approximately $300 \mathrm{~nm}$ thick to an interference pattern produced using a $325 \mathrm{~nm} \mathrm{He-Cd} \mathrm{laser.} \mathrm{This} \mathrm{pattern} \mathrm{was}$ chemically developed and then transferred to the silica substrate by reactive-ion etching the samples using a mixture of $\mathrm{CHF}_{3}$ and $\mathrm{O}_{2}$ gases; gratings had a pitch of $338 \mathrm{~nm}( \pm 1 \mathrm{~nm})$ and amplitudes in the range $15-40 \mathrm{~nm}$ [as determined from scanning electron micrographs-see Fig. 1(c)]. A $60 \mathrm{~nm}$ thick emissive organic layer of tris(8-hydroxyquinoline)aluminium $\left(\mathrm{Alq}_{3}\right)$ and a silver film were then sequentially added by thermal evaporation under high-vacuum conditions $\left(5 \times 10^{-7}\right.$ Torr $)$. A key point of our investigation was to probe the effect of the thickness of the silver film on the emission process. To do this we made four separate regions on two samples, the different regions differing only in their silver film thickness, ranging from $19 \pm 2 \mathrm{~nm}$ to $81 \pm 2 \mathrm{~nm}$, measured using a calibrated quartz crystal oscillator.

\section{B. Photoluminescence measurement technique}

To measure the angle-dependent photoluminescence, samples were mounted on a rotation stage and the $\mathrm{Alq}_{3}$ optically pumped through the silica substrate with light from a $410 \mathrm{~nm}$ diode laser. The spectrum of the resulting PL emitted through the silver film was recorded using a spectrometer/ CCD combination with a spectral resolution of $\sim 2 \mathrm{~nm}$, having first been passed through a narrow aperture to limit the collection angle to $1^{\circ}$. By rotating the sample while keeping the collection optics stationary, emission spectra could be recorded for a range of polar emission angles $(\theta)$. In order to keep the optical pumping conditions constant the angle of the pump beam relative to the sample was fixed throughout each experiment.

\section{RESULTS}

Figure 3 shows PL spectra from a corrugated sample with a silver film thickness of $55 \mathrm{~nm}$ for polar emission angles of $15^{\circ}, 20^{\circ}$, and $26^{\circ}$. A number of sharp emission features may be observed in these spectra (note that these data are presented using a logarithmic intensity scale), including a marked increase in intensity for an emission angle of $26^{\circ}$, at which the peak wavelength is $508 \mathrm{~nm}$. It should be noted that these emission features were only observed when the emission was collected with a polarizer set to pass TM polarized light and were not evident when the polarizer was set to pass TE polarized light. Also shown in Fig. 3 is the emission spectrum from a planar control sample, also acquired at an emission angle of $26^{\circ}$. Emission from the corrugated sample is seen to be far stronger than that from the planar sample; in particular, the emission from the corrugated struc-

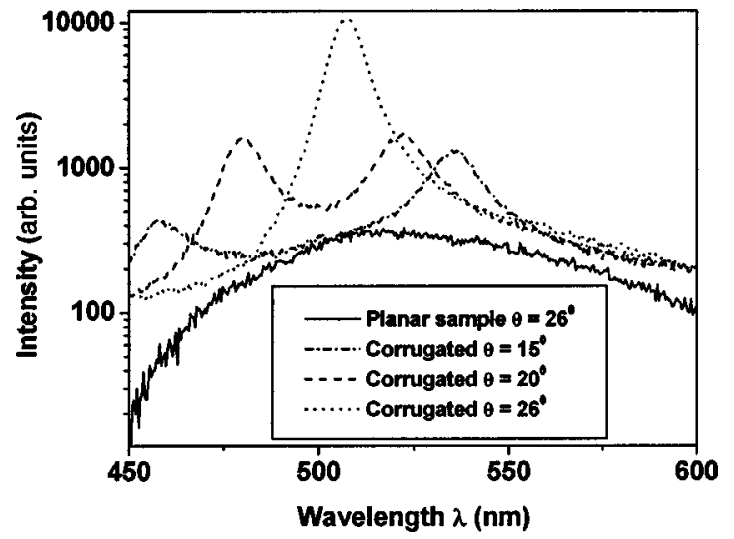

FIG. 3. Photoluminescence spectra of light emitted through a corrugated $55 \mathrm{~nm}$ thick silver film, and a $55 \mathrm{~nm}$ thick planar control film.

ture at a wavelength of $508 \mathrm{~nm}$ and an emission angle of $26^{\circ}$ is over 30 times stronger than that from the planar control structure.

\section{A. Identifying the modes in the PL spectra}

In order to identify the modes responsible for the emission features observed in data such as those shown in Fig. 3, a dispersion map was constructed using PL spectra taken over a range of emission angles. These data were converted from intensity as a function of wavelength and emission angle (as they were recorded) to functions of angular frequency and in-plane wave vector, thus facilitating the assembly of an experimentally derived dispersion map. One such dispersion map is shown in Fig. 4, where dark regions indicate strong emission. Comparing these data, Fig. 4, with the area enclosed within the dashed lines shown in the dispersion

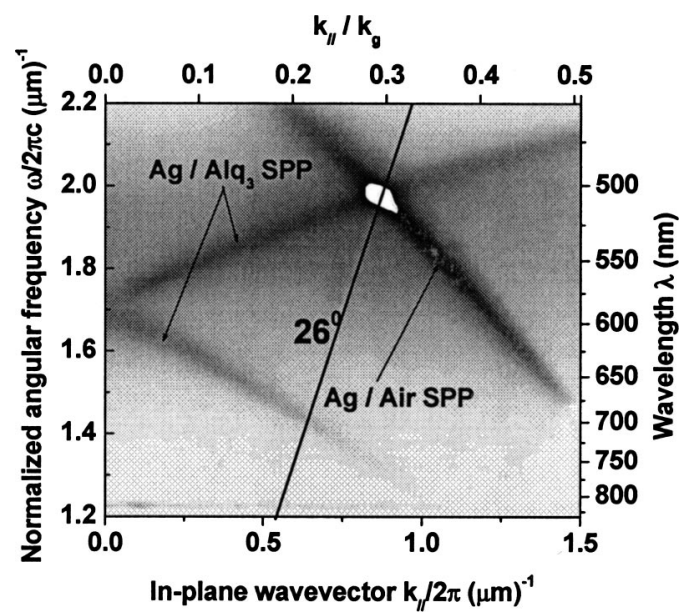

FIG. 4. Dispersion map constructed from photoluminescence (PL) emission spectra for a sample with a $55 \mathrm{~nm}$ thick corrugated silver film. The $z$ axis represents intensity and is on a log scale with dark regions indicating strong emission. The line labeled $26^{\circ}$ corresponds to the PL emission spectrum measured at a polar emission angle $(\theta)$ of $26^{\circ}$. In order to see the modal features the intensity at the crossing-point is overexposed. 
schematic, Fig. 1(b), we see that the sharp peaks in the emission spectra can be ascribed to the SPP modes associated with the metal/organic and metal/air interfaces. This conclusion is further supported by the observation that the PL of these modes is TM polarized.

There are two aspects of the data shown in Fig. 4 that should be noted. First, the region of strongest emission, seen in Fig. 4 at a wavelength of $508 \mathrm{~nm}$ and an angle of $26^{\circ}$, corresponds to the crossing point of the scattered SPP modes associated with the metal/air and metal/ $\mathrm{Alq}_{3}$ interfaces. Second, one can see that the emission associated with the metal/ organic SPP is in general weaker than that associated with the metal/air SPP. This is very surprising since as we saw above (Fig. 2), a far greater fraction of an emitter's power is coupled to the metal/organic SPP than the metal/air SPP (a factor of at least 100:1). The reason the metal/organic SPP is only weakly seen in the experiment can be described as follows. There are two ways in which this mode can be scattered to produce emitted light. ${ }^{29}$ First it may be scattered by the microstructure at the metal/organic interface. The light will then be attenuated by propagation through the metal before emerging as light into the far field. Second the microstructure at the metal/air boundary may scatter the mode. In this case it is the field associated with the metal/organic SPP that is attenuated across the metal. In both cases the attenuation is much the same so that these two routes have similar amplitude, let us call them $A_{\mathrm{SPP} \text { org }}^{\text {org }}$ and $A_{\mathrm{SPP} \text { org }}^{\text {air }}$ Importantly, recent work has shown that these two routes are out of phase, ${ }^{29}$ there is thus cancellation, the resulting intensity being $\left|A_{\mathrm{SPP} \text { org }}^{\text {org }}-A_{\mathrm{SPP} \text { org }}^{\text {air }}\right|^{2}$. This cancellation must be very substantial since our data, Fig. 4, indicates the intensity of emission mediated by the two SPP modes to be comparable in intensity whereas the power coupled from the emitters to the metal/air SPP is 100 times weaker than that coupled to the metal/organic SPP, Fig. 2.

\section{B. Emission in the crossing region}

The emission features seen in Fig. 4 show that SPP mediated emission occurs not only at the SPP crossing point, but over a range of frequencies and emission angles, provided they fall within the $\mathrm{Alq}_{3}$ emission spectrum. From the data shown in Fig. 4 it is not obvious whether more than the simple addition of the power associated with the scattered metal/organic and scattered metal/air SPPs is needed to explain the high value of the PL at the $\omega, k_{\|}$value at which the modes coincide (the crossing point). We now examine the strength of that emission at the crossing point to see if some extra mechanism, such as grating assisted SPP cross coupling, is needed to explain the observations.

In order to establish the strength of the PL at the crossing point it was first necessary to determine the strength of the direct emission from the individual SPP modes. This was achieved by tracking the features in the emission spectra as they evolved with emission angle and recording the peak intensity and wavelength associated with them. This process was carried out for the SPP modes associated with both the metal/organic and metal/air interfaces; the results are shown in Fig. 5. From these data we can measure the emitted inten-
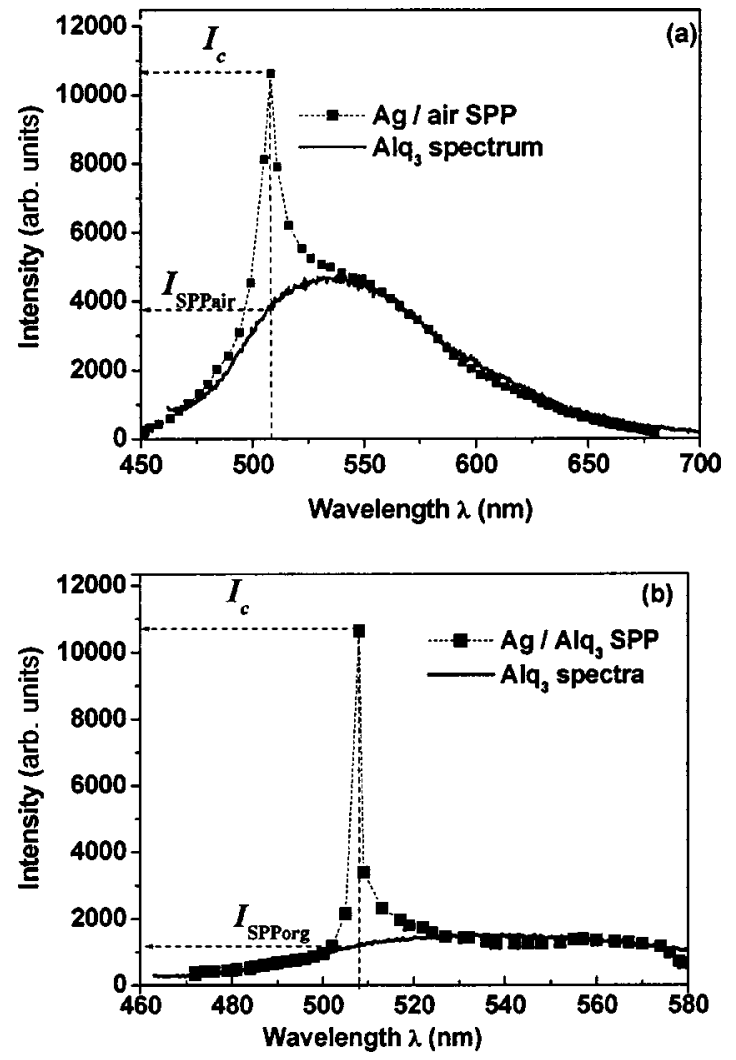

FIG. 5. Peak value of the photoluminescence emission associated with the SPP at (a) the $\mathrm{Ag} /$ air and (b) the $\mathrm{Ag} / \mathrm{Alq}{ }_{3}$ interfaces as one tracks the dispersion of each feature. The data shown are for a sample with a silver thickness of $55 \mathrm{~nm}$.

sity at the crossing point $I_{C}$. We can also infer the intensity mediated by the SPP modes associated with the metal/air and metal/organic interfaces would have at the same $\omega, k_{\|}$point ( $I_{\mathrm{SPP} \text { air }}$ and $I_{\mathrm{SPP} \text { org }}$, respectively) by fitting a normalized PL spectrum obtained from a planar $\mathrm{Alq}_{3}$ film to the background spectrum in both of the plots shown in Fig. 5. From the data shown in Fig. 5, obtained from a sample with a silver film thickness of $55 \mathrm{~nm}, I_{C}, I_{\mathrm{SPP} \text { org }}$, and $I_{\mathrm{SPP} \text { air }}$ were found to be $10620( \pm 210), 1175( \pm 100)$, and $3975( \pm 125)$, respectively. At first sight this would seem to imply that the simple addition of the emitted intensity from the two emission routes is not sufficient to explain the intensity measured at the crossing point since $I_{C}(=10620)>I_{\mathrm{SPP} \text { org }}$ $+I_{\mathrm{SPP} \text { air }}(=5150)$. However, before such a conclusion can be drawn a more careful analysis needs to be undertaken.

\section{DISCUSSION}

When considering how the light mediated by these SPP modes contributes to the emitted intensity at the crossing point we need to bear in mind that emission mediated by the two SPP modes is driven by the same source; the two routes are thus coherent. In fact, as we noted above, the situation is even more complex than this since the SPP mode associated with the metal/organic interface has two routes by which it may couple to radiation by scattering from the two corrugations. There are thus three coherent pathways to consider. 


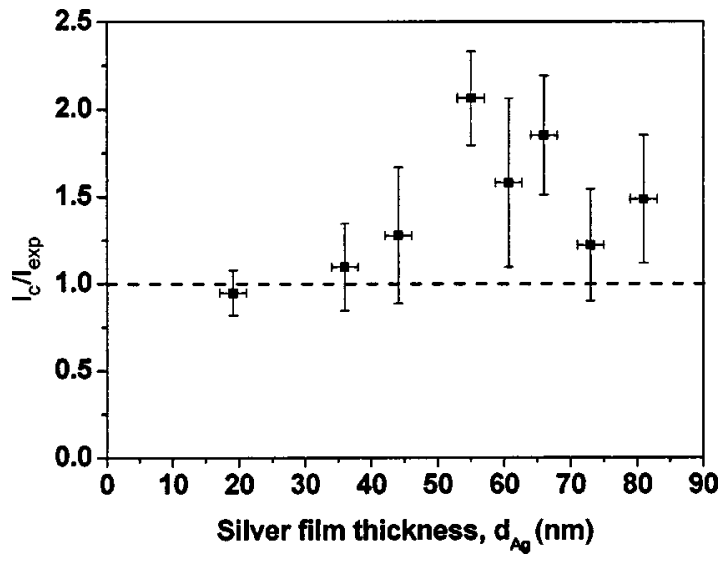

FIG. 6. The ratio of the observed intensity at the crossing point $I_{C}$ to the expected intensity $I_{\text {exp }}$.

We have already denoted the amplitude resulting from the metal/organic SPP scattered by corrugation at the metal/ organic interface as $A_{\mathrm{SPP} \text { org }}^{\mathrm{org}}$ and that resulting from the metal/air interface as $A_{\mathrm{SPP} \text { org }}^{\text {air }}$ let us further denote the amplitude of the light mediated by the SPP mode associated with the metal/air interface as $A_{\mathrm{SPP}}$ air. We now have to consider the relative phase of these different pathways. Let us consider first the metal/air SPP. Coupling from the source to this SPP mode proceeds via the far field since the wave vector of this SPP mode is less than that of a photon in the organic layer (the medium in which the emitter resides). There will thus be $a+\pi / 2$ phase change incurred as the far field is scattered by the corrugation to produce an evanescent wave and another $+\pi / 2$ phase change as this evanescent wave excites the SPP resonance. Coupling out will incur the same two phase changes so that the net phase change is $2 \pi(\equiv 0 \pi)$. For the metal/organic SPP, coupling from the source proceeds via the near field so that without the need to produce an evanescent wave by scattering there is just a phase change of $+\pi / 2$ needed to excite the SPP resonance. Coupling out of the SPP resonance incurs a phase change of $+\pi / 2$ and scattering to light another $+\pi / 2$. In addition, and as noted in Sec. III A, when this last scattering step takes place from the metal/organic interface an additional phase change of $+\pi$ is incurred. ${ }^{29}$ Taking all three pathways into account the resulting amplitude can thus be written as $A_{\mathrm{SPP} \text { air }}+\left(A_{\mathrm{SPP} \text { org }}^{\text {org }}-A_{\mathrm{SPP} \text { org }}^{\text {air }}\right) e^{i \pi / 2}$. We have no way to access $A_{\mathrm{SPP} \text { org }}^{\text {org }}$ and $A_{\mathrm{SPP} \text { org }}^{\text {air }}$ separately; let us simply denote their difference as $A_{\mathrm{SPP} \text { org }}$. The expected intensity $I_{\text {exp }}$ will thus be given by

$$
I_{\mathrm{exp}}=\left|A_{\mathrm{SPP} \text { air }}+A_{\mathrm{SPP} \text { org }} e^{i \pi / 2}\right|^{2}=I_{\mathrm{SPP} \text { air }}+I_{\mathrm{SPP} \text { org }},
$$

that is, it is given by the simple sum one anticipates in the absence of any interference effects.

In Fig. 6 we show the ratio $I_{C} / I_{\exp }$ as a function of silver film thickness. From these data we see that the observed intensity at the crossing point exceeds that expected on the basis of the contributions from the individual modes for all but the thinnest silver films. SPP cross coupling seems the most plausible mechanism by which this may occur. We fur-

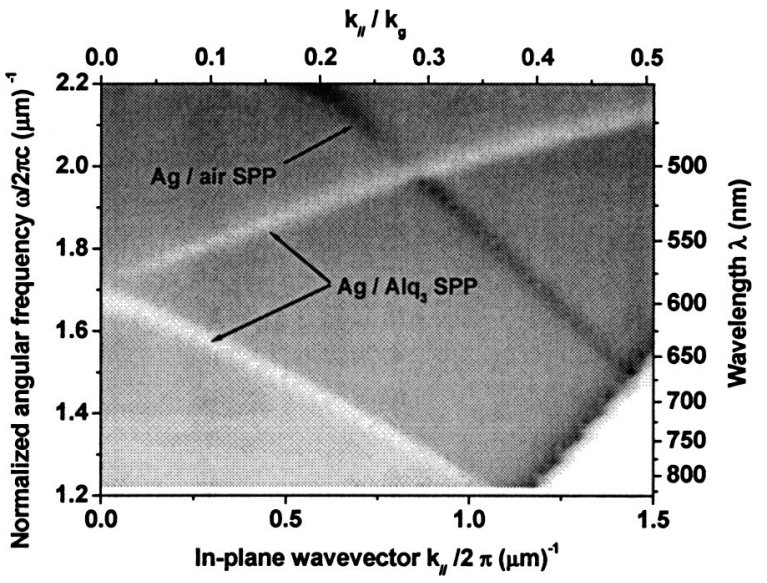

FIG. 7. TM polarized transmittance data in the form a dispersion map. The sample had a silver film thickness of $55 \mathrm{~nm}$.

ther note that that there appears to be a maximum in the ratio $I_{C} / I_{\exp }$ for silver film thicknesses around $60 \mathrm{~nm}$. Can SPP cross coupling be used to explain this aspect of the data as well?

It might be expected that if SPP cross coupling is taking place then the interaction between the two SPP modes might also lead to anticrossing in the SPP dispersion. ${ }^{7}$ There is no evidence for this in the dispersion data based on photoluminescence (Fig. 4) but as a check we also determined the dispersion from transmittance data. ${ }^{30}$ Transmittance measurements were taken as a function of both wavelength and angle of incidence, converted to transmittance data as a function of frequency and in-plane wave vector, and plotted to form a dispersion diagram, Fig. 7. Comparing this plot with the PL dispersion map shown in Fig. 4 for the $55 \mathrm{~nm}$ thick silver film, it may be seen that the features previously identified as being associated with the $\mathrm{Ag} /$ air and $\mathrm{Ag} / \mathrm{Alq}_{3} \mathrm{SPP}$ modes are also evident in transmission. Examination of Fig. 7 shows no evidence of such anti-crossing where the two modes intersect. Closer inspection of Fig. 7 shows a small stop band to be evident (both in Figs. 4 and 7) where counter propagating metal/organic SPP modes cross (at $\omega / 2 \pi c$ $=1.7 \mu \mathrm{m}$ and $\left.k_{\|} / 2 \pi=0\right)$. This stop band arises from the same scattering process as is required for an interaction (anticrossing) between the two different SPP modes at their crossing point. However, since the anticrossing involves modes from opposite sides of the metal we expect this to be a weaker effect than the stop band which involves modes on the same side of the metal. Given that the stop band we see is a very small one it is not altogether surprising that we see no evidence of an anticrossing in the dispersion data for the crosscoupled SPP modes. It would be interesting to explore whether more strongly modulated samples show such anticrossing can be produced; it would also be fascinating to see whether such changes might produce stronger emission based on SPP cross coupling.

We next wished to explore the reason for the seemingly greater effect of cross coupling (higher values of $I_{C} / I_{\exp }$ ) for film thicknesses around $60 \mathrm{~nm}$. We found it instructive to look at the ratios $I_{\mathrm{SPP} \text { air }} /\left(I_{\mathrm{SPP} \text { org }}+I_{\mathrm{SPP} \text { air }}\right)$ and $I_{\mathrm{SPP} \text { org }} /\left(I_{\mathrm{SPP} \text { org }}+I_{\mathrm{SPP} \text { air }}\right)$ as a function of silver film thick- 


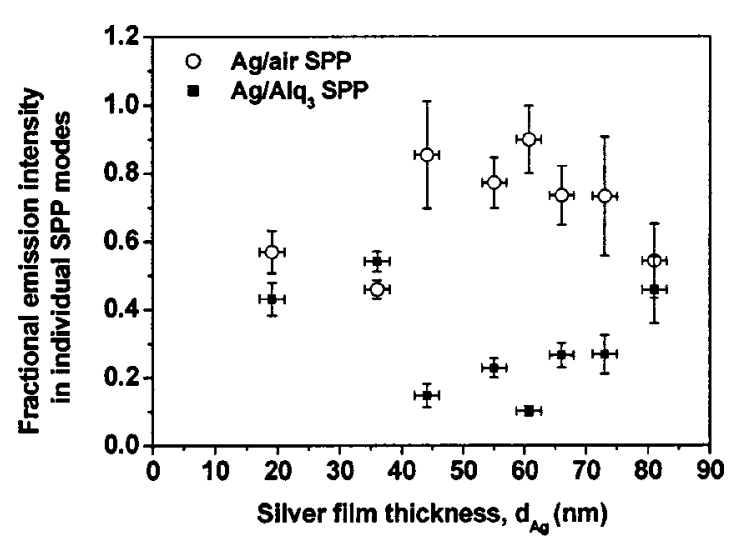

FIG. 8. Relative intensity of the emission seen via the two SPP modes as a function of silver film thickness.

ness, the results are shown in Fig. 8. We see that for small values of the silver film thickness, less than $40 \mathrm{~nm}$, the strength of the two pathways is roughly the same. As remarked above, this is despite the fact that an emissive source such as an $\mathrm{Alq}_{3}$ exciton in the light-emitting layer couples to the SPP associated with the metal/organic interface with a probability that is more than 100 times that of coupling to the SPP mode associated with the metal/air interface. For thicker films, around $60 \mathrm{~nm}$, there is a marked difference in the relative strength of the emission associated with the two SPP modes, that associated with the metal/air SPP being significantly stronger. Finally, for the thickest film measured, $80 \mathrm{~nm}$, the intensities are once again similar. The observation that the metal/air SPP dominates for silver films around $60 \mathrm{~nm}$ thickness is reminiscent of the optimum film thickness found for coupling incident plane waves to SPP at a metal/air interface using the Kretschmann-Raether ${ }^{31}$ prism coupling arrangement; we now investigate this possibility further.

We can identify two factors that need to be considered when trying to understand the change in PL emission intensity mediated by the two SPP modes that occurs with varying metal thickness. We need to consider how the emitters couple to the two SPP modes and we need to look at how these modes are in turn coupled to emitted light.

First let us consider the SPP associated with the metal/ organic interface. Emissive sources couple strongly to this mode via their near field and the strength of this coupling is largely independent of the metal film thickness, see Fig. 2. For the thinnest metal films there is a small change in the probability that an emitter will lose its energy to this mode since the nature of such a mode changes as the metal film thickness is reduced, ${ }^{24}$ and the field associated with the mode samples the air half space. As discussed above, there are two routes by which this mode can be scattered to produce emitted light; they suffer similar attenuation in getting through the metal and are out of phase with each other. The net consequence is that emission via the metal/organic SPP mode will both be weak (compared to the power coupled to the mode), and is expected to fall exponentially with increasing metal film thickness.

Second let us consider the SPP mode associated with the metal/air interface; for this mode things are rather different.
Here coupling between an emitter and this SPP mode proceeds via the far field of the emitter since the wave vector of the metal/air SPP is less than that of a photon in the organic layer. Once excited this SPP mode can be scattered by the microstructure at the metal/air interface to produce emitted light. This latter scattering process is largely independent of metal film thickness, but the strength of coupling from the emitter to this SPP mode falls exponentially with increasing metal film thickness (Fig. 2) as one might expect. We thus expect that the strength of emission mediated by both SPP routes will fall exponentially with increasing metal film thickness. The increase in the relative strength of the metal/ air SPP mode for film thicknesses around $60 \mathrm{~nm}$ (Fig. 8) cannot therefore be explained on the basis of the mechanisms considered so far; perhaps cross coupling of the SPPs is responsible. As noted above, the increase in the relative strength of the metal/air SPP mode for film thicknesses around $60 \mathrm{~nm}$ is reminiscent of the optimum metal film thickness in Kretschmann-Raether prism coupling to SPPs using attenuated total reflection: let us explore this possibility in more detail.

In the cross-coupling scheme the SPP mode associated with the metal/organic interface can couple to the SPP mode associated with the metal/air interface when scattering off the microstructure is such as to allow their wave vector mismatch to be overcome [see Eq. (1)]. The field associated with the metal/organic SPP falls exponentially (at least to a first approximation) across the metal film where it can be scattered by the microstructure so as to couple to the metal/air SPP. A phase change of $\pi / 2$ is incurred as the evanescent field of the metal/organic SPP excites the metal/air SPP. ${ }^{29}$ The reverse process can also occur, that is, the SPP associated with the metal/air SPP (that has been generated by scattering of the metal/organic SPP) can in turn be scattered so as to couple to the metal/organic SPP. This return route incurs another $\pi / 2$ phase change so that the original SPP field and that generated by this return route are out of phase. If the amplitude of this return field could be made equal to the initial source field then we have cancellation. One might think that such a thing cannot be accomplished since the field will suffer exponential decay on both passages through the metal film. However, by exciting the SPP associated with the metal/air interface the amplitude of the field is enhanced ${ }^{32}$ so that the amplitude of the return field can indeed equal that of the source, provided the thickness of the film is chosen so that the field enhancement is balanced by exponential decay of the field across the metal. When the resulting cancellation occurs power will have been effectively transferred from the metal/organic SPP to the metal/air SPP. This line of reasoning is at least consistent with the observations (Fig. 8) and provides further support for the idea that SPP cross coupling is taking place.

In summary, we have investigated the emission of light through a metal film, focussing particularly on the role of the two SPP modes associated with metal/organic and metal/air interfaces of such structures. We have shown that when emission mediated by both of these modes emerges in the same direction and at the same wavelength there are a 
number of subtle effects that come into play, notably the interference of different emission pathways. By considering these possibilities we have established that our experimental data are at least consistent with the idea of SPP cross coupling. However we have also shown that such cross coupling is a relatively weak phenomenon-perhaps due to the modest amplitudes of the grating structures we have used. The phenomenon of SPP cross coupling may have consequences for devices such as surface-emitting organic light-emitting diodes; it also provides a fascinating arena in which to ex- plore some of the subtle physics associated with surface plasmon polaritons.

\section{ACKNOWLEDGMENTS}

The authors would like to thank J. A. E. Wasey for useful discussions. This work was supported by the Materials Domain of the UK MoD Corporate Research Program; the authors are also grateful to the EPSRC for financial support.
${ }^{1}$ L. S. Hung, C. W. Tang, M. G. Mason, P. Raychaudhuri, and J. Madathi, Appl. Phys. Lett. 78, 544 (2001).

${ }^{2}$ S. Han, X. Feng, Z. H. Lu, D. Johnson, and R. Wood, Appl. Phys. Lett. 82, 2715 (2003).

${ }^{3}$ P. A. Hobson, J. A. E. Wasey, I. Sage, and W. L. Barnes, IEEE J. Sel. Top. Quantum Electron. 8, 378 (2002).

${ }^{4}$ P. A. Hobson, S. Wedge, J. A. E. Wasey, I. Sage, and W. L. Barnes, Adv. Mater. (Weinheim, Ger.) 14, 1393 (2002).

${ }^{5}$ J. M. Lupton, B. J. Matterson, I. D. W. Samuel, M. J. Jory, and W. L. Barnes, Appl. Phys. Lett. 77, 3340 (2000).

${ }^{6}$ D. K. Gifford and D. G. Hall, Appl. Phys. Lett. 81, 4315 (2002).

${ }^{7}$ I. Pockrand, Opt. Commun. 13, 311 (1975).

${ }^{8}$ M. G. Weber and D. L. Mills, Phys. Rev. B 32, 5057 (1985).

${ }^{9}$ S. R. J. Brueck, V. Diadiuk, T. Jones, and W. Lenth, Appl. Phys. Lett. 46, 915 (1985).

${ }^{10}$ J. Giergiel, C. E. Reed, J. C. Hemminger, and S. Ushioda, Phys. Rev. B 36, 3052 (1987).

${ }^{11}$ S. Ushioda, J. E. Rutledge, and R. M. Pierce, Phys. Rev. Lett. 54, 224 (1985).

${ }^{12}$ R. W. Gruhlke, W. R. Holland, and D. G. Hall, Phys. Rev. Lett. 56, 2838 (1986).

${ }^{13}$ T. W. Ebbesen, H. J. Lezec, H. F. Ghaemi, T. Thio, and P. A. Wolff, Nature (London) 391, 667 (1998).

${ }^{14}$ N. Bonod, S. Enoch, L. Li, E. Popov, and M. Nevière, Opt. Express 11, 482 (2003).

${ }^{15}$ A. Köck, K. Beinstingl, K. Berthold, and E. Gornik, Appl. Phys.
Lett. 52, 1164 (1988).

${ }^{16}$ A. Köck, E. Gornik, M. Hauser, and K. Beinstingl, Appl. Phys. Lett. 57, 2327 (1990).

${ }^{17}$ W. L. Barnes, J. Lightwave Technol. 17, 2170 (1999).

${ }^{18} \mathrm{~J}$. Vuckovic, M. Loncar, and A. Scherer, IEEE J. Quantum Electron. 36, 1131 (2000).

${ }^{19}$ N. E. Hecker, R. A. Hopfel, and N. Sawaki, Physica E (Amsterdam) 2, 98 (1998).

${ }^{20}$ N. E. Hecker, R. A. Hopfel, N. Sawaki, T. Maier, and G. Strasser, Appl. Phys. Lett. 75, 1577 (1999).

${ }^{21}$ D. K. Gifford and D. G. Hall, Appl. Phys. Lett. 80, 3679 (2002).

${ }^{22}$ J. R. Sambles, G. W. Bradbery, and F. Z. Yang, Contemp. Phys. 32, 173 (1991).

${ }^{23}$ D. Sarid, Phys. Rev. Lett. 47, 1927 (1981).

${ }^{24}$ K. R. Welford and J. R. Sambles, J. Mod. Opt. 35, 1467 (1988).

${ }^{25}$ A. E. Craig, G. A. Olson, and D. Sarid, Opt. Lett. 8, 380 (1983).

${ }^{26}$ Y. Kuwamura, M. Fukui, and O. Tada, J. Phys. Soc. Jpn. 52, 2350 (1983).

${ }^{27}$ J. A. E. Wasey and W. L. Barnes, J. Mod. Opt. 47, 725 (2000).

${ }^{28}$ W. L. Barnes, J. Mod. Opt. 45, 661 (1998).

${ }^{29}$ I. R. Hooper and J. R. Sambles, Phys. Rev. B 67, 235404 (2003).

${ }^{30}$ M. G. Salt and W. L. Barnes, Opt. Commun. 166, 151 (1999).

${ }^{31}$ E. Kretschmann and H. Raether, Z. Naturforsch. A 23, 2135 (1968).

${ }^{32}$ W. H. Weber and G. W. Ford, Opt. Lett. 6, 122 (1981). 